\title{
Circulating Levels of Interleukin-6 and Interleukin-10, But Not Tumor Necrosis Factor-Alpha, as Potential Biomarkers of Severity and Mortality for COVID-19: Systematic Review with Meta-analysis
}

\author{
Wanvisa Udomsinprasert $^{1}$ (D) - Jiraphun Jittikoon ${ }^{1} \cdot$ Sermsiri Sangroongruangsri ${ }^{2} \cdot$ Usa Chaikledkaew $^{2,3}$
}

Received: 12 August 2020 / Accepted: 22 October 2020 / Published online: 31 October 2020

(C) Springer Science+Business Media, LLC, part of Springer Nature 2020

\begin{abstract}
Purpose Cytokine storm, an uncontrolled overproduction of inflammatory cytokines contributing to an aberrant systemic inflammatory response, is a major pathological feature of acute respiratory distress syndromes being severe manifestations of COVID-19, thus highlighting its potential as a biomarker and therapeutic target for COVID-19. We aimed to determine associations of circulating levels of inflammatory cytokines with severity and mortality of COVID-19 by systematic review and metaanalysis.

Methods A comprehensive literature search in electronic databases consisting of PubMed, Scopus, and Cochrane Library and in a hand searching of reference lists from inception to July 31, 2020, was performed using the following search terms: COVID-19, interleukin (IL)-6, IL-10, and tumor necrosis factor-alpha (TNF- $\alpha$ ). Mean difference (MD) from individual studies was pooled using a random-effects model. Quality assessment, publication bias, meta-regression, subgroup, and sensitivity analyses were performed.

Results A total of 6212 COVID-19 patients from 24 eligible studies were included. Compared with non-severe COVID-19 patients, systemic levels of IL-6 and IL-10, but not TNF- $\alpha$, were significantly elevated in severe COVID-19 patients (MD = 18.63, 95\% CI: 10.91, 26.35, $P<0.00001 ; \mathrm{MD}=2.61,95 \% \mathrm{CI}: 2.00,2.32, P<0.00001$; respectively). For COVID-19 mortality, circulating levels of IL-6, IL-10, and TNF- $\alpha$ were found to be significantly increased in non-survivors when compared with survivors $(\mathrm{MD}=57.82,95 \% \mathrm{CI}: 10.04,105.59, P=0.02 ; \mathrm{MD}=4.94,95 \% \mathrm{CI}: 3.89,6.00, P<0.00001 ; \mathrm{MD}=5.60,95 \% \mathrm{CI}$ : 4.03, 7.17, $P<0.00001$; respectively).

Conclusion Circulating levels of IL-6 and IL-10 might have great potential as biomarkers for the disease severity and mortality in COVID-19 patients.
\end{abstract}

Keywords COVID-19 cytokine storm $\cdot$ interleukin- $6 \cdot$ interleukin-10 $\cdot$ tumor necrosis factor-alpha

Supplementary Information The online version contains supplementary material available at https://doi.org/10.1007/s10875-02000899-z.

Wanvisa Udomsinprasert

wanvisa.udo@mahidol.ac.th

1 Department of Biochemistry, Faculty of Pharmacy, Mahidol University, 447 Sri-Ayudhaya Road, Rajathevi, Bangkok 10400, Thailand

2 Social and Administrative Pharmacy Division, Department of Pharmacy, Faculty of Pharmacy, Mahidol University, Bangkok 10400, Thailand

3 Mahidol University Health Technology Assessment (MUHTA) Graduate Program, Mahidol University, Bangkok 10400, Thailand

\section{Introduction}

Coronavirus disease 2019 (COVID-19), a newly emerging acute respiratory disease, is caused by severe acute respiratory syndrome coronavirus 2 (SARS-CoV-2) initially detected in Wuhan, China [1]. Although the epidemiology and pathophysiology of COVID-19 are constantly being clarified, until now the disease has been rapidly spread worldwide and has continued to pose a major therapeutic challenge $[1,2]$. Given the ongoing pandemic and no effective standard of care, some patients with severe COVID-19 develop pneumonia, acute respiratory distress syndrome (ARDS), and multi-organ dysfunction being leading causes of death [3]. From this, it is important to note that the early identification of respiratory symptoms associated with ARDS would be of paramount 
importance in not only hindering the need for mechanical ventilation but also declining mortality. This point has accelerated a need to identify sensitive and specific biomarkers of progression toward severe and fatal forms, which would open the opportunity to develop effective preventive strategies and/ or therapeutic options.

Of various risk factors known to persuade the developmental and progressive COVID-19, cytokine storm, a hyperinflammation by which cytokines are released, has been increasingly recognized as a keystone event driving ARDS progression in COVID-19 patients [4]. It is becoming apparent that disruption in cytokine storm holds great promise as a potential therapeutic intervention for COVID19. In this regard, there is a wide spectrum of immuneactive molecules including interleukins (IL) and tumor necrosis factor-alpha (TNF- $\alpha$ ) proposed as contributors to the development of cytokine storm [5]. Among ILs, IL-6 as a multifunctional mediator of inflammation is widely thought to play a pivotal role in SARS-CoV-2-induced cytokine storm and to participate in interstitial pneumonia and ARDS observed in severe COVID-19 [6]. In the acute phase of inflammation and infection, IL-6 is produced by immune cells-especially T-helper 17 (TH17) cells in COVID-19 patients [7]. In addition to this, the production of this cytokine is also increased by TNF- $\alpha$ as a pyrogen cytokine released from immune cells in response to chronic inflammatory and autoimmune diseases, suggesting IL-6 as a downstream effector of TNF- $\alpha$ [8]. Besides IL-6, IL10 is synthesized from regulatory T cells and even TH 1 cells and has been reportedly implicated in immunoregulation and inflammation [9], thereby highlighting the power of IL-10 to influence immune and inflammatory responses in the context of COVID-19. Indeed, a number of clinical studies have unveiled that circulating IL-6 levels were elevated in COVID-19 patients, particularly in those with severe stage and were positively associated with the severity and mortality of COVID-19 [10-33], commensurate with circulating levels of IL-10 and TNF- $\alpha[12,14,15$, $17,18,20,21,24,28,29]$. Based on these previous findings, alterations in circulating levels of IL-6, IL-10, and TNF- $\alpha$ have been hypothesized to be biomarkers of disease severity and mortality for COVID-19.

Although the previous meta-analysis has linked circulating IL-6 levels to the severity of COVID-19 [34], until now no attempt has been made to capture the breadth of systemic levels of IL- 6 along with IL-10 and TNF- $\alpha$ related to the disease severity and mortality. Accordingly, we conducted an up-to-date systematic review and metaanalysis of the currently available literature regarding cytokine storm in COVID-19 to evaluate whether circulating levels of IL-6, IL-10, and TNF- $\alpha$ have any influence on poor clinical outcomes and reduced survival rates in patients with COVID-19.

\section{Methods}

This systematic review was conducted in adherence to the recommendations outlined in the Cochrane Handbook [35] and the Preferred Reporting Items for Systematic Reviews and Meta-Analyses (PRISMA) guidelines [36].

\section{Search Strategy}

A systematic literature search of electronic databases including PubMed, Scopus, and the Cochrane library was performed to identify relevant studies from inception and up to July 31 , 2020, without language restrictions using the following search terms: "coronavirus 2019," "COVID-19," "2019-nCoV," "SARS-CoV-2," "interleukin-6," "interleukin-10," "tumor necrosis factor-alpha." The detailed search strategies are provided in the online supplement.

After acquiring preliminary database search results, 2 authors (W.U. and J.J.) independently reviewed the titles and abstracts of articles to exclude studies that did not address the research question of interest. To further identify relevant studies, the reference list of each included study was comprehensively searched. Afterwards, the same 2 authors (W.U. and J.J.) independently assessed the full text of the remaining articles and determined whether it contained relevant and complete information using the predefined inclusion and exclusion criteria, as detailed below. Furthermore, the bibliographies of the selected articles and review articles on the topic were searched for additional studies that may have been missed. Any discrepancies regarding study inclusion between authors were resolved through discussion among the reviewers (W.U., J.J., S.S., and U.C.). In the case of duplicate studies from the same cohort, we included data from the most recent comprehensive report.

\section{Definition of Clinical Outcomes}

Clinical outcomes evaluated in our study included severe and non-severe COVID-19, in addition to mortality. Severe COVID-19 was defined by individual studies and comprised any of the following: COVID-19 patients who suffer from shortness of breath, respiratory frequency $\geq 30 / \mathrm{min}$, blood oxygen saturation $\leq 93 \%$, the ratio of arterial oxygen partial pressure $\left(\mathrm{PaO}_{2}\right.$ in $\left.\mathrm{mmHg}\right)$ to fractional inspired oxygen $\left(\mathrm{FiO}_{2}\right.$ expressed as a fraction $)<300$, and/or lung infiltrates $>50 \%$ within 24-48 h. Non-severe COVID-19 was defined by patients without pneumonia or cases of mild pneumonia. We accepted definitions defined by individual studies.

\section{Inclusion and Exclusion Criteria}

Relevant studies were included in this meta-analysis if they met the following inclusion criteria: (1) human studies 
including adult or pediatric subjects with a known diagnosis of COVID-19; (2) the studies being case-control, cross-sectional, or cohort designs; (3) the studies reporting on circulating levels of IL-6, IL-10, and TNF- $\alpha$ in COVID-19 patients dichotomized into severe/critical or non-severe groups, as well as non-survivors or survivors. Meanwhile, the exclusion criteria were the following: (1) review articles and other systematic reviews or meta-analyses; (2) non-human studies including cell culture and animal models; (3) no measure of those cytokines available; (4) the severity and mortality of COVID-19 not reported; (5) incomplete data on clinical outcome measures (circulating levels of IL-6, IL-10, and TNF- $\alpha$ represented as either mean and standard deviation (SD) or median and interquartile range (IQR) with specified clinical outcomes not provided).

\section{Data Extraction}

Data were extracted from the eligible studies by 2 investigators independently (W.U. and J.J.) using a preformatted data collection form, and any disagreements regarding qualitative and quantitative data collection were resolved through discussion by other authors (S.S. and U.C.). Extracted data included the following: study characteristics - primary author, year of publication, country of origin, types of study design, the number of patients with severe and non-severe COVID-19, the number of survivors and non-survivors; patient characteristics - mean age and percentage of male participants; assay characteristics - types of assay used for measurement on circulating levels of IL-6, IL-10, and TNF- $\alpha$; outcome measures - circulating levels of IL-6, IL-10, and TNF- $\alpha$ displayed as either mean and SD or median and IQR in patients with severe and non-severe COVID-19, in addition to those patients divided into non-survivors and survivors. When such data were not explicitly reported, they were requested from the authors through personal contacts for clarification or further information, wherever possible.

\section{Quality Assessment}

Two authors (W.U. and J.J.) independently evaluated the methodological quality of included studies using the Newcastle-Ottawa scale for observational studies including cohort and case-control designs [37]. The following domains were assessed: adequacy of definition of cases, representativeness of the defined cases, criteria used for selection of controls, comparability of cases and controls, and method of ascertainment of exposure. The Newcastle-Ottawa scores were defined as high (7-9 stars), moderate (4-6 stars), or low (0-3 stars). Any discrepancies in the assessment of study quality were resolved by consensus agreement by other authors (S.S. and U.C.).

\section{Data Synthesis}

Data from each continuous outcome were pooled and presented as mean difference (MD), and summaries of data were evaluated using a random-effects model, as a result of the anticipated variability in methodologies between studies. For some analyses, the median was converted to mean, IQR was converted to $\mathrm{SD}$, and the range was transformed to $\mathrm{SD}$, according to previously published studies [38, 39]. Heterogeneity between studies was executed using Cochran's $Q$ test with a 0.10 level of significance and $I^{2}$ statistic test with the cutoffs of $<30 \%, 30 \%-59 \%, 60 \%-75 \%$, and $>75 \%$ indicating low, moderate, substantial, and considerable heterogeneity, respectively [40]. When significant heterogeneity was evident from an $I^{2}$ value of $>30 \%$, sources of heterogeneity were investigated using meta-regression and subgroup analyses based on the following categorical variables: average age at baseline $(<60$ or $\geq 60$ years), gender (mean percentage of male participants $<60 \%$ or $\geq 60 \%$ ), study region (Asia, Europe, or North America), study design (retrospective cohort, retrospective case-control, or prospective cohort studies), and cytokine assay (chemiluminescent immunoassay (CLIA), flow cytometry (FCM), or enzyme-linked immunosorbent assay (ELISA)). To determine if the overall results were robust, a sensitivity analysis was further carried out by sequential omission of individual studies. Publication bias was assessed using the visual examination of funnel plots and quantitatively using Egger's test. When the funnel plot became asymmetrical, and Egger's test reported a $P$ value < 0.05 , publication bias was assumed to exist. All statistical analyses were accomplished by Review Manager (RevMAN) version 5.3 (The Nordic Cochrane Centre, The Cochrane Collaboration, Denmark) and STATA software version 15.1 (STATA, College Station, TX, USA), and a $P$ value of $<0.05$ was considered statistically significant.

\section{Results}

\section{Systematic Search Results}

The flow diagram detailing the studies' selection process is depicted in Fig. 1. Out of 2180 citations, 24 articles with a total of 6212 COVID-19 patients met our predefined inclusion and exclusion criteria. Of the studies deemed possibly relevant and screened against inclusion criteria, the main reasons for exclusion were the unavailable data for the assessment of severity and mortality for COVID-19 and the lack of data on circulating levels of inflammatory cytokines including IL-6, IL-10, and TNF- $\alpha$. The descriptive characteristics of the included studies are summarized in Table 1. From the 24 included studies, 17 studies reported on blood levels of inflammatory cytokines such as IL- 6 , IL-10, or TNF- $\alpha$ in severe and non- 
Table 1 Baseline characteristics of the included studies investigating blood levels of inflammatory cytokines in COVID-19 patients

\begin{tabular}{|c|c|c|c|c|c|c|c|c|c|}
\hline Authors & Year & Country & Study design & Group & $\begin{array}{l}\text { Number } \\
(n)\end{array}$ & Age (years) & $\begin{array}{l}\text { Gender }(\% \\
\text { male })\end{array}$ & $\begin{array}{l}\text { Cytokine } \\
\text { assay }\end{array}$ & $\begin{array}{l}\text { Study } \\
\text { quality }\end{array}$ \\
\hline \multicolumn{10}{|l|}{ Severity of COVID-19 } \\
\hline \multirow[t]{2}{*}{ Burian et al. [10] } & \multirow[t]{2}{*}{2020} & \multirow[t]{2}{*}{ Germany } & \multirow[t]{2}{*}{ Retrospective cohort study } & Severe & 12 & $64.9 \pm 16.6$ & $64.6 \%$ & \multirow[t]{2}{*}{ CLIA } & \multirow[t]{2}{*}{6} \\
\hline & & & & Non-severe & 25 & $59.0 \pm 17.1$ & $54.1 \%$ & & \\
\hline \multirow[t]{2}{*}{ Chen et al. [11] } & \multirow[t]{2}{*}{2020} & \multirow[t]{2}{*}{ China } & \multirow[t]{2}{*}{ Retrospective cohort study } & Severe & 27 & $73.8 \pm 14.0$ & $64.6 \%$ & \multirow[t]{2}{*}{ CLIA } & \multirow[t]{2}{*}{6} \\
\hline & & & & Non-severe & 21 & $52.8 \pm 14.2$ & $61.9 \%$ & & \\
\hline \multirow[t]{2}{*}{ Chi et al. [12] } & \multirow[t]{2}{*}{2020} & \multirow[t]{2}{*}{ China } & \multirow{2}{*}{$\begin{array}{l}\text { Retrospective case-control } \\
\text { study }\end{array}$} & Severe & 8 & $54.0 \pm 12.4$ & $63.0 \%$ & \multirow[t]{2}{*}{ CLIA } & \multirow[t]{2}{*}{8} \\
\hline & & & & Non-severe & 58 & $41.8 \pm 15.4$ & $55.2 \%$ & & \\
\hline Gao et al. [13] & 2020 & China & Retrospective cohort study & Severe & 15 & $45.2 \pm 7.7$ & $60.0 \%$ & CLIA & 6 \\
\hline & & & & Non-severe & 28 & 43.0. \pm 14.0 & $60.7 \%$ & & \\
\hline Han et al. [14] & 2020 & China & Retrospective case-control & Severe & 60 & $59.3 \pm 14.4$ & $48.8 \%$ & FCM & 7 \\
\hline & & & study & Non-severe & 42 & $58.3 \pm 12.6$ & $47.6 \%$ & & \\
\hline He et al. [15] & 2020 & China & Retrospective cohort study & Severe & 33 & $53.9 \pm 12.5$ & $55.0 \%$ & CLIA & 6 \\
\hline & & & & Non-severe & 60 & $44.5 \pm 12.5$ & $52.0 \%$ & & \\
\hline Liu et al. [16] & 2020 & China & Retrospective cohort study & Severe & 7 & $52.0 \pm 14.7$ & $57.1 \%$ & CLIA & 7 \\
\hline & & & & Non-severe & 44 & $41.9 \pm 12.3$ & $63.7 \%$ & & \\
\hline Liu et al. [17] & 2020 & China & Retrospective cohort study & Severe & 66 & $56.4 \pm 44.7$ & $47.8 \%$ & ELISA & 6 \\
\hline & & & & Non-severe & 11 & $39.1 \pm 27.1$ & $9.1 \%$ & & \\
\hline Lv et al. [18] & 2020 & China & Retrospective cohort study & Severe & 239 & $63.4 \pm 41.5$ & $49.0 \%$ & CLIA & 6 \\
\hline & & & & Non-severe & 115 & $54.0 \pm 42.0$ & $50.4 \%$ & & \\
\hline Ma et al. [19] & 2020 & China & Retrospective cohort study & Severe & 20 & $66.6 \pm 9.6$ & $50.0 \%$ & CLIA & 6 \\
\hline & & & & Non-severe & 17 & $61.0 \pm 4.9$ & $58.9 \%$ & & \\
\hline Qin et al. [20] & 2020 & China & Retrospective cohort study & Severe & 286 & $60.3 \pm 13.4$ & $54.2 \%$ & CLIA & 6 \\
\hline & & & & Non-severe & 166 & $52.0 \pm 15.5$ & $48.2 \%$ & & \\
\hline Wan et al. [21] & 2020 & China & Retrospective cohort study & Severe & 21 & $61.3 \pm 15.6$ & $52.4 \%$ & FCM & 8 \\
\hline & & & & Non-severe & 45 & $43.1 \pm 13.2$ & $53.9 \%$ & & \\
\hline Wang et al. [22] & 2020 & China & Retrospective cohort study & Severe & 12 & $62.9 \pm 9.89$ & $57.1 \%$ & FCM & 6 \\
\hline & & & & Non-severe & 33 & $63.1 \pm 14.1$ & $48.4 \%$ & & \\
\hline Wang et al. [23] & 2020 & China & Retrospective cohort study & Severe & 50 & $56.6 \pm 12.8$ & $44.0 \%$ & CLIA & 6 \\
\hline & & & & Non-severe & 115 & $45.0 \pm 20.7$ & $43.5 \%$ & & \\
\hline Wang et al. [24] & 2020 & China & Retrospective cohort study & Severe & 8 & $5.1 \pm 4.5$ & $75.0 \%$ & CLIA & 6 \\
\hline & & & & Non-severe & 35 & $6.9 \pm 1.8$ & $60.0 \%$ & & \\
\hline Wu et al. [25] & 2020 & China & Retrospective cohort study & Severe & 63 & $51.9 \pm 14.26$ & $63.3 \%$ & CLIA & 7 \\
\hline & & & & Non-severe & 77 & & & & \\
\hline Xie et al. [26] & 2020 & China & Retrospective cohort study & Severe & 24 & $70.9 \pm 18.8$ & $54.2 \%$ & CLIA & 6 \\
\hline & & & & Non-severe & 38 & $59.5 \pm 16.3$ & $36.8 \%$ & & \\
\hline Mortality of COVID-19 & & & & & & & & & \\
\hline Crespo et al. [27] & 2020 & Spain & Prospective cohort study & Non-survivors & 8 & $74.6 \pm 5.3$ & $62.5 \%$ & CLIA & 8 \\
\hline & & & & Survivors & 8 & $72.6 \pm 4.2$ & $87.5 \%$ & & \\
\hline Huang et al. [28] & 2020 & China & Retrospective cohort study & Non-survivors & 4 & $38.3 \pm 11.4$ & $50.0 \%$ & CLIA & 6 \\
\hline & & & & Survivors & 27 & $37.0 \pm 10.2$ & $45.0 \%$ & & \\
\hline Luo et al. [29] & 2020 & China & Retrospective cohort study & Non-survivors & 201 & $69.7 \pm 11.9$ & $66.2 \%$ & CLIA & 6 \\
\hline & & & & Survivors & 817 & $56.3 \pm 17.9$ & $47.5 \%$ & & \\
\hline Mikami et al. [30] & 2020 & US & Retrospective cohort study & Non-survivors & 806 & $75.3 \pm 14.7$ & $59.9 \%$ & CLIA & 8 \\
\hline & & & & Survivors & 2014 & $61.3 \pm 17.8$ & $56.0 \%$ & & \\
\hline Quartuccio et al. [31] & 2020 & Italy & Prospective cohort study & Non-survivors & 6 & $68.8 \pm 9.4$ & $83.3 \%$ & CLIA & 6 \\
\hline & & & & Survivors & 18 & $65.8 \pm 8.2$ & $66.7 \%$ & & \\
\hline Tu et al. [32] & 2020 & China & Retrospective cohort study & Non-survivors & 25 & $71.4 \pm 12.6$ & $76.0 \%$ & CLIA & 8 \\
\hline & & & & Survivors & 149 & $49.9 \pm 18.7$ & $40.3 \%$ & & \\
\hline Zhou et al. [33] & 2020 & China & Retrospective cohort study & Non-survivors & 54 & $69.4 \pm 9.9$ & $70.0 \%$ & CLIA & 8 \\
\hline & & & & Survivors & 137 & $51.6 \pm 9.7$ & $59.0 \%$ & & \\
\hline
\end{tabular}

CLIA, chemiluminescent immunoassay; COVID-19, coronavirus disease 2019; ELISA, enzyme-linked immunosorbent assay; FCM, flow cytometry

severe COVID-19 patients, and 7 remaining studies provided data on the involvement of circulating levels of those cytokines in the mortality of COVID-19. All of these were observational studies: 20 were retrospective cohort studies; 2 were retrospective case-control studies; and 2 were prospective cohort studies. Of note, COVID-19 patients included in the systematic review and meta-analysis come from 3 continents (Asia $(n=20)$, Europe $(n=3)$, and North America $(n=1))$ and 5 countries (China $(n=20)$, Germany $(n=1)$, Spain $(n=1)$, Italy $(n=1)$, and US $(n=1))$. The mean age of participants was 56.62 years, and the mean percentage of male patients was $56 \%$. In regard to the cytokine method, most studies 
employed CLIA $(n=20)$, followed by FCM $(n=3)$ and $\operatorname{ELISA}(n=1)$.

\section{Quality Assessment}

Details on the methodological quality of the included studies based on the Newcastle-Ottawa scale are provided in Table S1. Quality scores of the included studies ranged from 6 to 8 stars (median 6 stars), from which the total score for each study is summarized in Table 1.

\section{IL-6 Levels in COVID-19 Patients with Different Groups Based on Disease Severity and Mortality}

\section{Comparison Between Patients with Severe and Non-severe COVID-19}

For the disease severity categorized into severe and non-severe groups, 17 out of 24 studies [10-26] reporting on circulating IL-6 levels in patients with and without severe COVID-19 and including a total of 1881 COVID-19 patients were meta-analyzed. Compared with patients with non-severe COVID-19, circulating IL-6 levels were found to be significantly elevated in those with severe COVID-19 (MD $=18.63,95 \%$ CI: 10.91 ,
26.35, $P<0.00001, I^{2}=95 \%$ ) (Fig. 2a). Despite the considerable heterogeneity, visual inspection of the funnel plot (Fig. S1) and Egger's test $(P=0.06)$ suggest no convincing evidence of publication bias in this meta-analysis.

To identify the potential sources of considerable heterogeneity, meta-regression and subgroup analyses according to age, gender, study region, study design, and cytokine assay were further undertaken. As detailed in Table 2, meta-regression analysis revealed that the heterogeneity could be explained by a difference in study design. Consistent with this finding, subgroup analysis by study design denoted a significant increase in circulating IL-6 levels in severe COVID-19 patients recruited in retrospective case-control studies (MD $=13.21,95 \% \mathrm{CI}$ : 5.98 to 20.45, $P=0.0003)$ and no heterogeneity $\left(I^{2}=0 \%\right)$ (Fig. 2b). In terms of age, gender, study region, and cytokine assay, although circulating IL-6 levels remained significantly escalated in patients with severe conditions, considerable heterogeneity existed (Fig. S2a-d). All findings derived from meta-regression and subgroup analyses indicate that the main source of heterogeneity may be applicable to study design. To confirm the robustness of the model, a sensitivity analysis was additionally executed. After simultaneously eliminating each study from the model, a
Fig. 1 Schematic diagram for study selection of the relevant articles

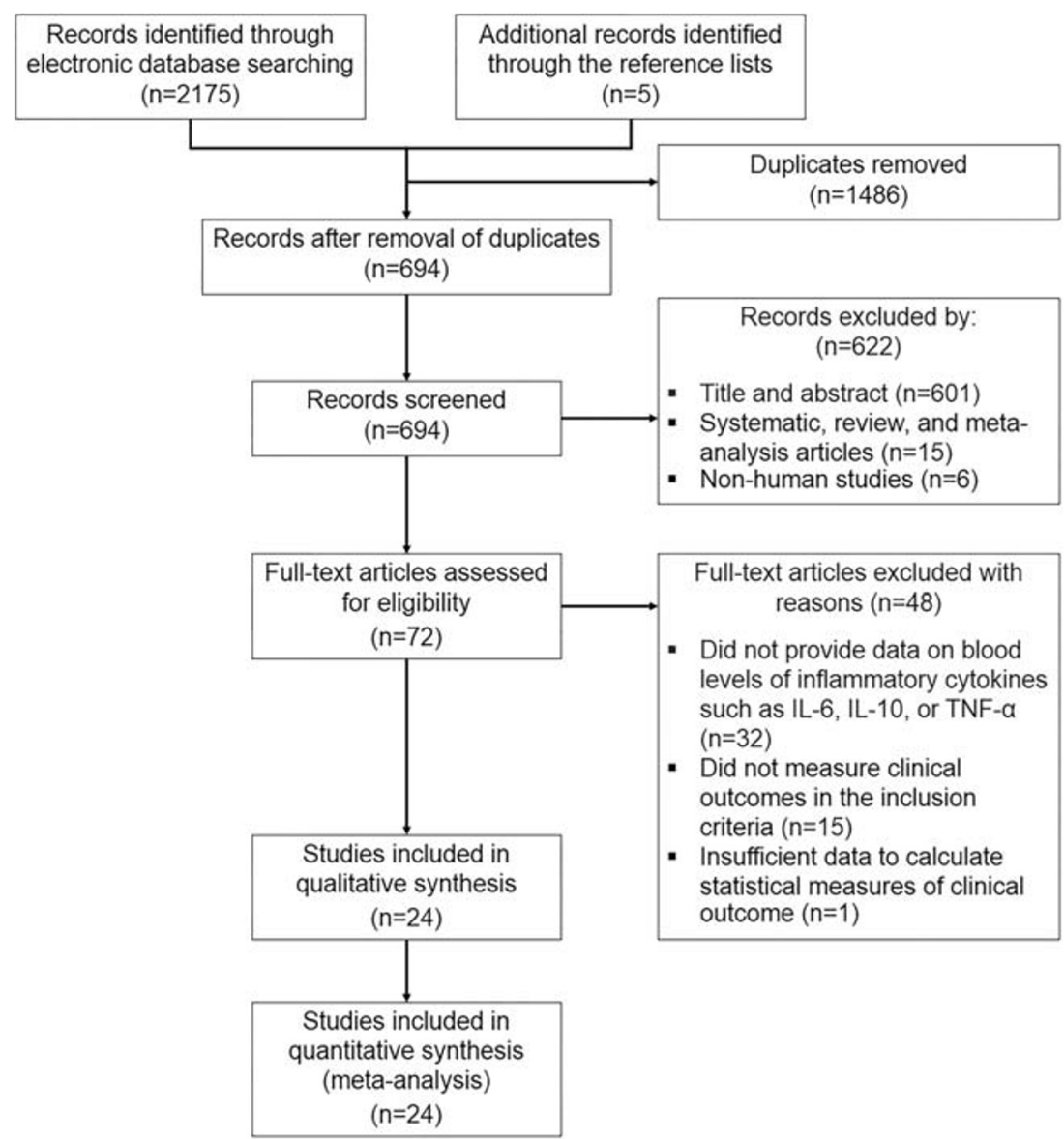


Fig. 2 Forest plot showing circulating IL-6 levels in COVID19 patients with different groups based on disease severity and mortality. a Meta-analysis of circulating IL-6 levels in severe and non-severe COVID-19 patients. $\mathbf{b}$ Subgroup analysis based on study design for circulating IL-6 levels in severe and non-severe COVID19 patients. c Meta-analysis of circulating IL-6 levels in nonsurvivors and survivors

\section{a IL-6 levels in severe and non-severe COVID-19}

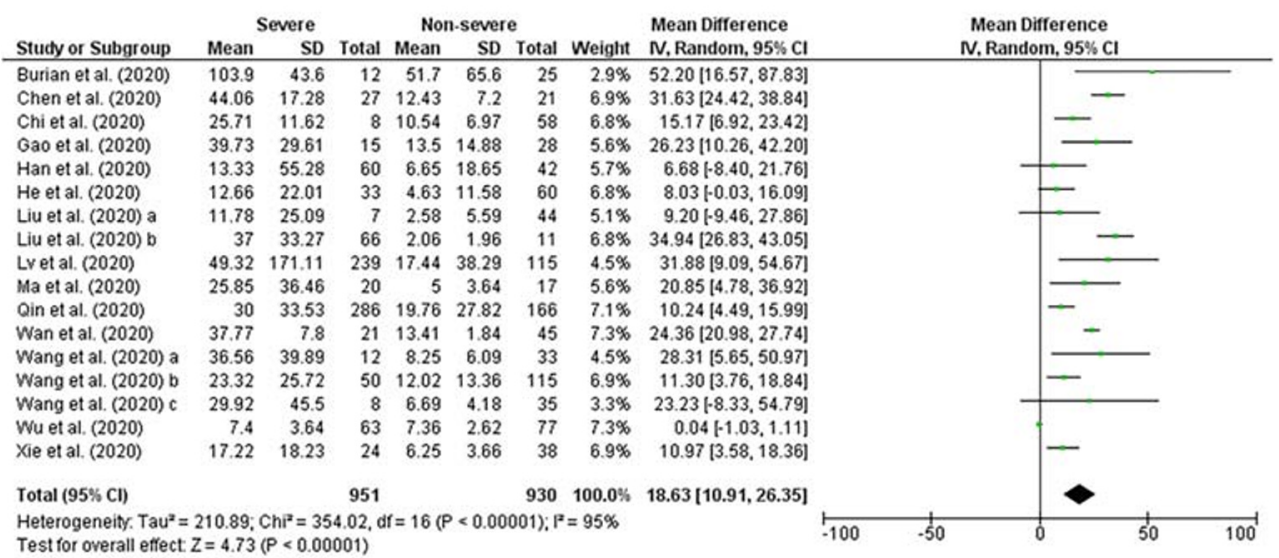

b Subgroup analysis by study design

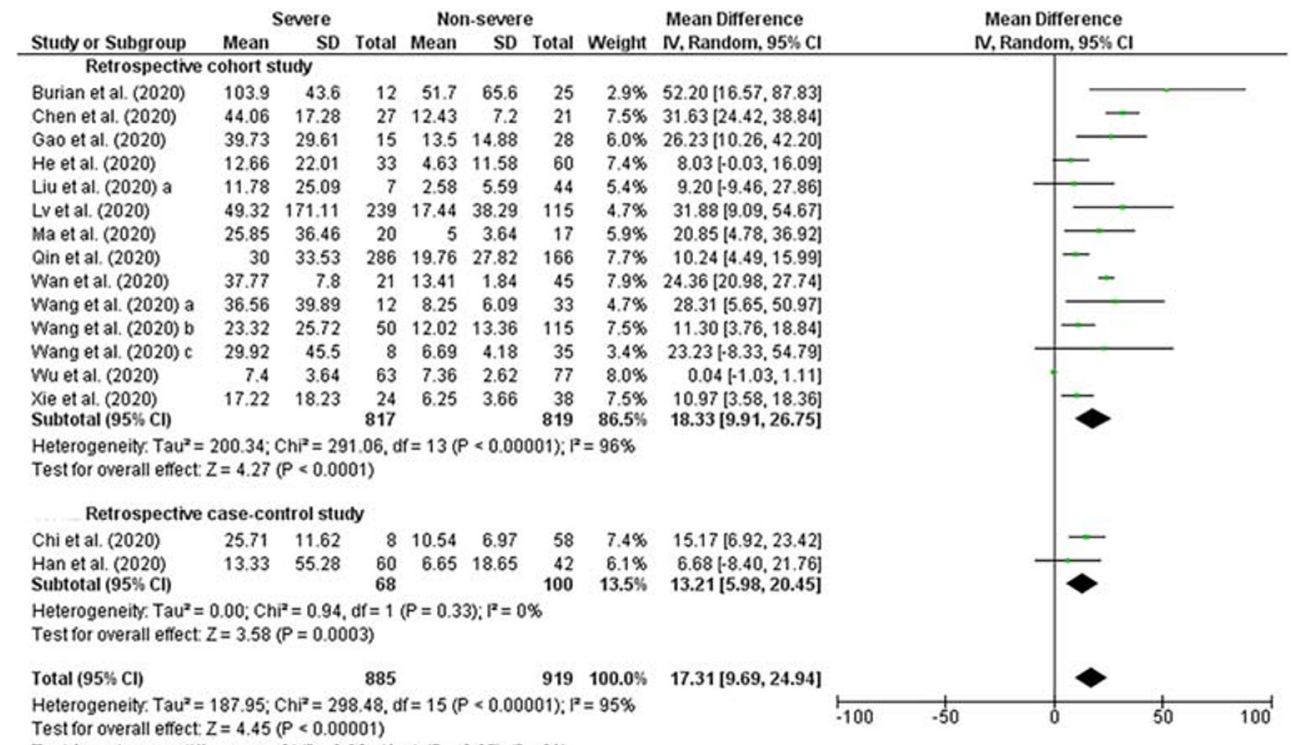

Test for subaroup differences: $\mathrm{Chi}^{2}=0.82, \mathrm{df}=1(\mathrm{P}=0.37), \mathrm{P}^{\mathrm{P}}=0 \%$

\section{c IL-6 levels in non-survivors and survivors}

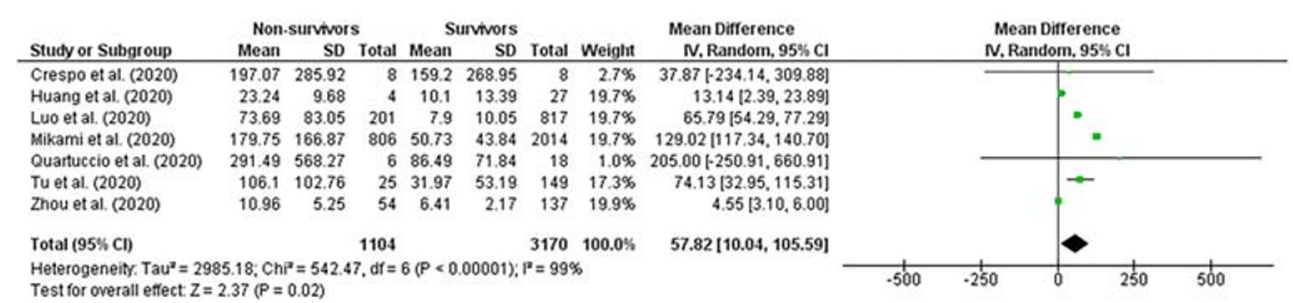

significant difference in circulating IL-6 levels between severe and non-severe cases remained.

\section{Comparison Between Non-survivors and Survivors}

In stratified meta-analysis with regard to mortality in the patients divided into non-survivors and survivors, a metaanalysis of 7 studies [27-33] providing evidence for circulating IL-6 levels in non-survivors and survivors in the context of
COVID-19 uncovered that 1104 non-survivors had significantly higher circulating IL-6 levels than 3170 survivors $(\mathrm{MD}=57.82,95 \% \mathrm{CI}: 10.04,105.59, P=0.02)$, albeit in the presence of heterogeneity $\left(I^{2}=99 \%\right)$ (Fig. $2 \mathrm{c}$ ). Due to the insufficient studies, assessment for publication bias was not accomplished.

The considerable heterogeneity was addressed by metaregression analysis showing that the overall pooled effect was unaffected by age, gender, study region, and study design 
Table 2 Meta-regression analysis for identifying the potential sources of heterogeneity

\begin{tabular}{|c|c|c|c|c|c|c|}
\hline \multirow[t]{2}{*}{ Covariates } & \multirow[t]{2}{*}{ Coefficient } & \multirow[t]{2}{*}{$\mathrm{SE}$} & \multirow[t]{2}{*}{$t$ test } & \multicolumn{2}{|l|}{$95 \% \mathrm{CI}$} & \multirow[t]{2}{*}{$P$ value } \\
\hline & & & & Lower & Upper & \\
\hline \multicolumn{7}{|l|}{ Severity of COVID-19 } \\
\hline \multicolumn{7}{|l|}{ IL-6 } \\
\hline Age & 11.47 & 8.21 & 1.40 & -6.03 & 28.98 & 0.18 \\
\hline Gender ( $\%$ male $)$ & 6.39 & 8.30 & 0.77 & -11.32 & 24.09 & 0.45 \\
\hline Study region & 34.57 & 23.54 & 1.47 & -15.61 & 84.74 & 0.16 \\
\hline Study design & 17.64 & 4.19 & 4.22 & 8.72 & 26.57 & 0.001 \\
\hline Cytokine method & 18.60 & 12.90 & 1.44 & -9.05 & 46.26 & 0.17 \\
\hline \multicolumn{7}{|c|}{ Mortality of COVID-19 } \\
\hline \multicolumn{7}{|l|}{ IL-6 } \\
\hline Age & 8.74 & 17.20 & 0.51 & -35.47 & 52.94 & 0.63 \\
\hline Gender ( $\%$ male $)$ & 67.82 & 32.38 & 2.09 & -15.41 & 151.06 & 0.09 \\
\hline Study region & 36.26 & 15.99 & 2.27 & -8.12 & 80.64 & 0.09 \\
\hline Study design & 69.29 & 33.15 & 2.09 & -15.94 & 154.51 & 0.09 \\
\hline Cytokine method & - & - & - & - & - & - \\
\hline \multicolumn{7}{|l|}{ TNF- $\alpha$} \\
\hline Age & 18.94 & 18.92 & 1.00 & -221.42 & 259.30 & 0.50 \\
\hline Gender ( $\%$ male $)$ & - & - & - & - & - & - \\
\hline Study region & 4.74 & 0.53 & 8.92 & 2.01 & 19.49 & 0.04 \\
\hline Study design & - & - & - & - & - & - \\
\hline Cytokine method & - & - & - & - & - & - \\
\hline
\end{tabular}

Values in italics denote statistical significance at the $P<0.05$ level

$C I$, confidence interval; COVID-19, coronavirus disease 2019; IL-6, interleukin-6; TNF- $\alpha$, tumor necrosis factoralpha; $S E$, standard error
(Table 2), which was attested by subgroup analyses based on those covariates (Fig. S3a-d). In the sensitivity analysis, removing a single study included in the pooled analysis did not affect the significant result demonstrating elevated circulating IL-6 levels in non-survivors.

\section{IL-10 Levels in COVID-19 Patients with Different Groups Based on Disease Severity and Mortality}

\section{Comparison Between Patients with Severe and Non-severe COVID-19}

In total, 9 studies $[12,14,15,17,18,20-22,24]$ reporting on circulating IL-10 levels in COVID-19 patients with severe ( $n=733)$ and non-severe conditions $(n=622)$ were included in this meta-analysis. As depicted in Fig. 3a, a significant increment in circulating IL-10 levels was observed in severe cases when compared with non-severe cases $(\mathrm{MD}=2.16$, 95\% CI: $2.00,2.32, P<0.00001)$ and the heterogeneity became invisible $\left(I^{2}=0 \%\right)$. In this meta-analysis, we did not assess for publication bias using a funnel plot, given the lack of sufficient studies.
Sensitivity analysis confirmed the robustness of the model because the significance remained after simultaneously omitting each study from the model.

\section{Comparison Between Non-survivors and Survivors}

Out of 24 studies, 2 studies [28, 29] reported on circulating IL-10 levels in COVID-19 patients who were either dead or alive. As demonstrated in Fig. 3b, circulating IL-10 levels were found to be elevated in non-survivors compared with survivors $(\mathrm{MD}=4.94$, 95\% CI: 3.89, 6.00, $P<0.00001)$, and the included studies appear to be homogeneous $\left(I^{2}=0 \%\right)$. Owing to less than 10 included studies, assessment for publication bias was not fulfilled.

\section{TNF-a Levels in COVID-19 Patients with Different Groups Based on Disease Severity and Mortality}

\section{Comparison Between Patients with Severe and Non-severe COVID-19}

Further meta-analysis of 8 studies $[12,14,15,17,18,20-22]$ investigating circulating TNF- $\alpha$ levels in severe $(n=725)$ and non-severe COVID-19 patients $(n=587)$ showed no 
a IL-10 levels in severe and non-severe COVID-19

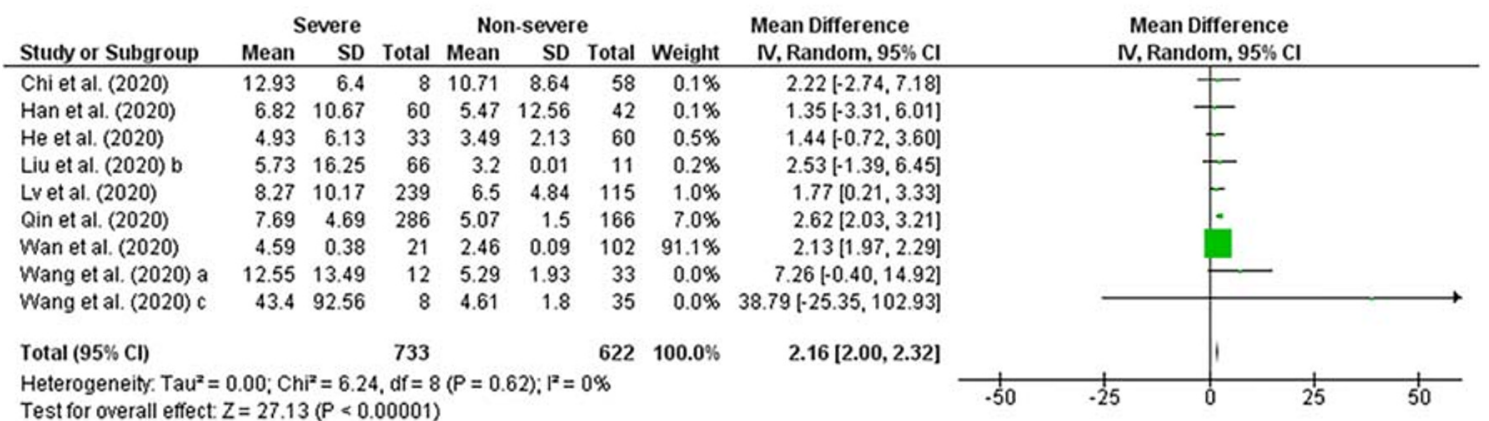

\section{b IL-10 levels in non-survivors and survivors}

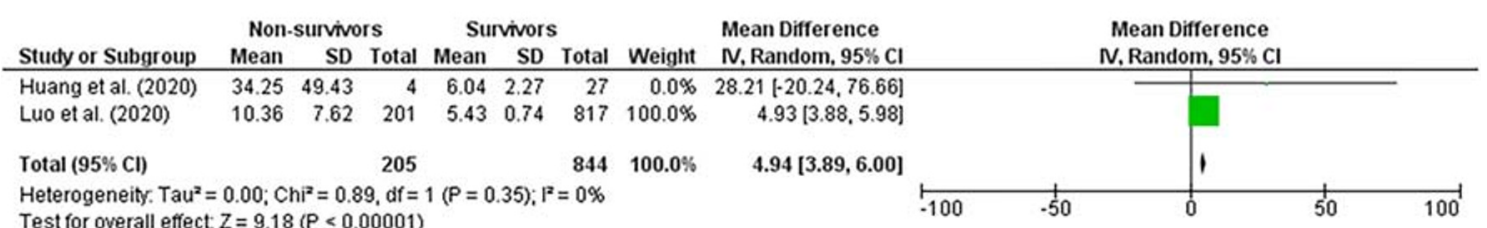

Fig. 3 Forest plot showing circulating IL-10 levels in COVID-19 patients with different groups based on disease severity and mortality. a Meta-analysis of circulating IL-10 levels in severe and non-severe COVID-19 patients. b Meta-analysis of circulating IL-10 levels in non-survivors and survivors

statistically significant difference in circulating TNF- $\alpha$ levels between groups (Fig. S4).

\section{Comparison Between Non-survivors and Survivors}

Aside from the disease severity, circulating TNF- $\alpha$ levels in non-survivors $(n=1011)$ and survivors $(n=2858)$ were evaluated in the 3 included studies [28-30]. As shown in Fig. 4a, there was a significant increase in circulating TNF- $\alpha$ levels in non-survivors when compared with survivors ( $\mathrm{MD}=5.60$, $95 \%$ CI: 4.03, 7.17, $P=0.0001)$. Despite this, substantial heterogeneity was found $\left(I^{2}=64 \%\right)$. As the minimum number of studies for assessment of publication bias ( $\geq 10$ studies) was unachievable, the test was not performed in this meta-analysis.

To elaborate on the heterogeneity, meta-regression analysis was carried out. The analysis showed the study region as a potential source of heterogeneity (Table 2). Likewise, subgroup analysis by study region revealed a marked elevation in circulating TNF- $\alpha$ levels in non-survivors - especially in the Asian patients (MD $=4.74,95 \%$ CI: 3.70, 5.78, $P<0.0001)$, without the heterogeneity $\left(I^{2}=0 \%\right)$ (Fig. 4b). After simultaneously eradicating each study from the model, sensitivity analysis displayed that an increase in circulating TNF- $\alpha$ levels in non-survivors compared with survivors remained statistically significant.

\section{Discussion}

Cytokine storm, the excessive production of inflammatory cytokines, has been well recognized as one of the most common causes of severe complications including ADRS-induced multi-organ failure leading to death in COVID-19 patients. It is noteworthy that targeting cytokines during the management of COVID-19 patients could improve clinical outcomes and reduce mortality. In support of this hypothesis, this metaanalysis of cumulative data derived from 24 studies has been focused on investigating the involvement of inflammatory cytokines including IL-6, IL-10, and TNF- $\alpha$ in the severity and mortality of COVID-19. Overall, our results demonstrated significant increases in circulating levels of IL-6 and IL-10 in both patients with severe COVID-19 and non-survivors, which confirm our notion that high circulating levels of inflammatory cytokines might have an important influence on poor clinical outcomes and reduced survival rates in COVID-19 patients.

As to their general biology, cytokines, produced by a variety of immune cells including macrophages, dendritic cells, natural killer cells, and adaptive $\mathrm{T}$ and $\mathrm{B}$ lymphocytes, are an essential part of the inflammatory process [41], in which an innate immune system is the first line of defensive mechanism against virus infection, subsequently triggering the expressions of pro-inflammatory cytokines including IL-1 and TNF- $\alpha$ [42]. Excessive production of those cytokines can cause an influx of various immune cells from the circulation into the site of infection with destructive effects on the damaged tissue including lung injury, one of the consequences of the cytokine storm that can progress into ADRS being a major cause of mortality in COVID-19 [43]. For this reason, the uncontrolled overproduction of inflammatory cytokines is considered to be one of the major contributing mediators of severe COVID-19 and mortality in the patients. This assumption has been partially addressed by a number of clinical 
a TNF- $\alpha$ levels in non-survivors and survivors

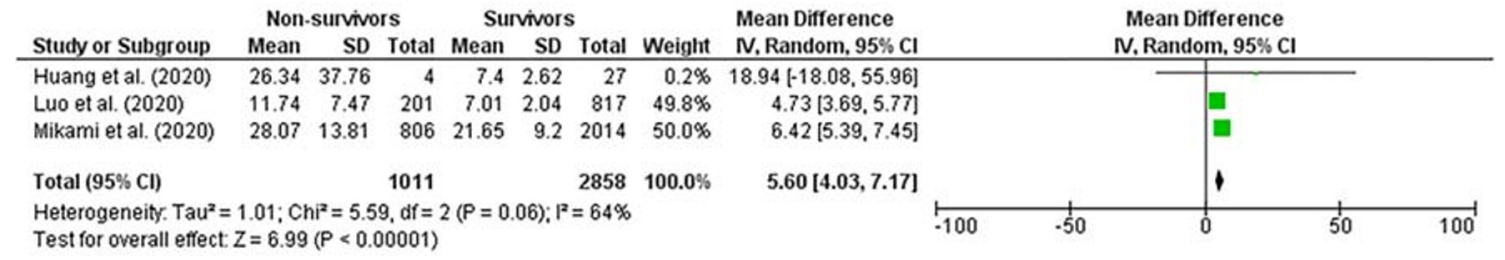

\section{b Subgroup by study region}

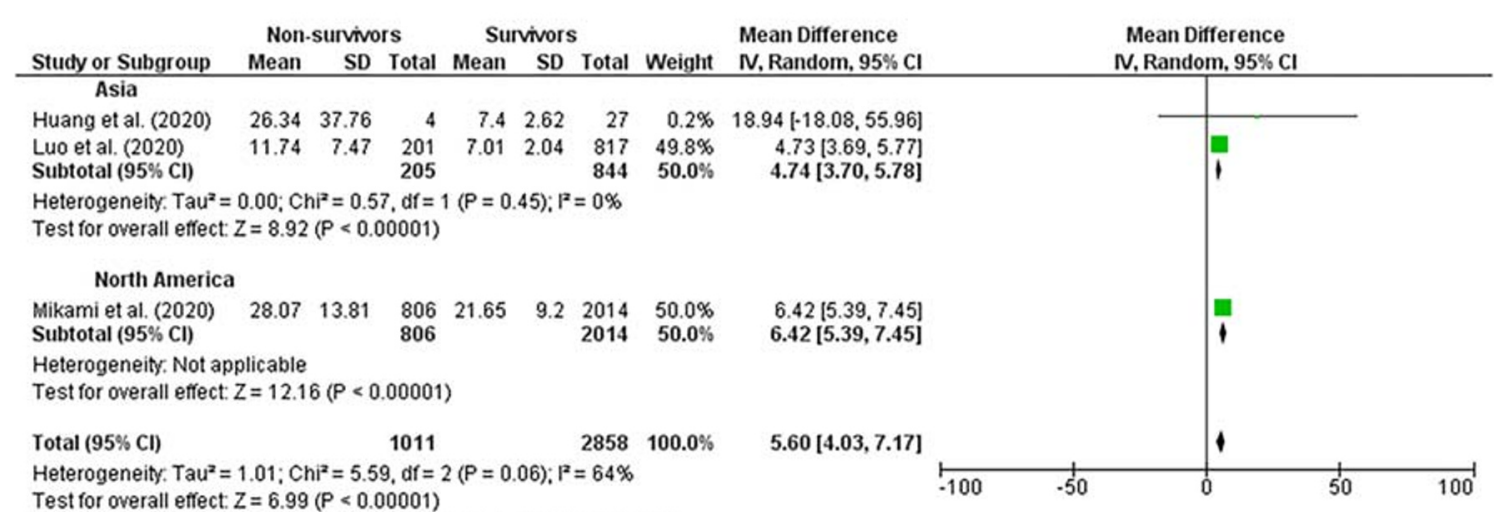

Fig. 4 Forest plot showing circulating TNF- $\alpha$ levels in COVID-19 patients with different groups based on disease mortality. a Meta-analysis of circulating TNF- $\alpha$ levels in non-survivors and survivors. b Subgroup

analysis based on study region for circulating TNF- $\alpha$ levels in nonsurvivors and survivors

studies, which denoted the significant associations between circulating levels of inflammatory cytokines, particularly IL6 and the severity and mortality of COVID-19 [10-13, 17-22, 26, 28-30, 32, 33]. More precisely, a previous meta-analysis [34] added another piece of supporting data, revealing that circulating IL-6 levels in severe COVID-19 patients was significantly higher than that in the non-severe patients. In line with the aforementioned findings, this meta-analysis uncovered that severe COVID-19 patients had significantly increased circulating IL-6 levels, as compared with those with non-severe COVID-19. Nevertheless, it may give a misleading result, due to the significant heterogeneity presented herein. This problem was overcome by meta-regression and subgroup analyses unveiling study design as an important source of heterogeneity, without any effect on the overall estimate confirmed by sensitivity analysis. Along with increased circulating levels of IL-6 in severe COVID-19, our subsequent meta-analysis revealed that its levels were significantly greater in non-survivors than that in survivors. However, this result should be interpreted with caution, because of the considerable heterogeneity. In an attempt to elaborate on the heterogeneity observed, meta-regression, subgroup, and sensitivity analyses were further undertaken; the results of which remained congruent with the overall meta-analysis, but the heterogeneity was evident. It should be noted that the heterogeneity could be explained by other confounding factors such as genetic polymorphism, COVID-19 treatment, or comorbidity that may influence circulating IL-6 levels. Unfortunately, subgroup analyses based on the above covariates were unachievable, given the unaffordability of those data in most included studies. Even though to date no meta-analyses reporting on the involvement of circulating IL-6 levels in mortality of COVID-19 were identified, previous results from a number of clinical studies [28-30,32,33] lend support to our significant finding regarding increased circulating IL-6 levels in non-survivors.

Aside from IL-6, IL-10 produced by immune cells responsible for an inflammatory response initiated the damaged tissue has been considered an additional molecule implicated in the severity and mortality of COVID-19. To address this postulation, our further meta-analysis depicted remarkably raised circulating IL-10 levels in severe COVID-19 patients compared with non-severe COVID-19 patients. Our main finding from this meta-analysis may help clarify the inconclusive results derived from several observational studies unveiling that circulating IL-10 levels were higher in severe cases than that in non-severe cases, but the difference is not statistically significant $[12,14,15,17,22,24]$. As regards IL-10 levels relevant to mortality, when 2 previous studies were considered, a study by Huang et al. [28] evinced no statistically significant difference in circulating IL-10 levels between non-survivors and survivors. Contrariwise, another previous study by Luo 
et al. [29] uncovered a significant elevation in circulating IL10 levels in non-survivors. In an endeavor to clarify its relevance to mortality in COVID-19 patients, we performed a meta-analysis of the abovementioned studies with contrasting results and also found that non-survivors exhibited considerably increased circulating IL-10 levels, as compared with survivors.

As an upstream of IL-6 in the cytokine cascade responsible for inflammatory and immune responses, it is not surprising that TNF- $\alpha$ has been proposed as a key cytokine in viral diseases - particularly COVID-19. Confirming its potential utility as a biomarker for COVID-19 severity, accumulating data derived from previous studies showing higher circulating levels of TNF- $\alpha$ in severe COVID-19 patients than that in non-severe COVID-19 patients are often corroborated [12, $14,17,18,20,22]$; however, the finding is still controversial $[12,15,21]$. These discordant results have led to uncertainty whether elevated circulating TNF- $\alpha$ has any influence on poor outcomes and reduced survival rates in COVID-19 patients. In our additional meta-analyses, there was no significant difference in circulating TNF- $\alpha$ levels between severe and nonsevere cases, while COVID-19 patients who died were observed to have substantially greater its circulating levels than those who survive. In spite of the considerable heterogeneity observed, finding from subgroup analysis showed that the study region was the potential cause of heterogeneity, but the significant result was unchanged, which was confirmed by sensitivity analysis. In light of all the above considerations, it appears that increased circulating levels of inflammatory cytokines including IL-6 and IL-10 might reflect poor clinical outcomes and reduced survival rates of COVID-19 patients. For the result of no significant difference in circulating TNF- $\alpha$ levels between severe and non-severe COVID-19 patients, the possible explanation may be attributed to TNF- $\alpha$ action as an upstream transcriptional regulator for inflammatory and immune cascades responsible for not only ADRS in the context of COVID-19 but also a variety of pathological conditions. Supporting this, several previous studies yielded the conflicting results of circulating levels of IL-1 $\beta$, a pro-inflammatory cytokine-like TNF- $\alpha$, in severe and non-severe COVID-19 patients $[12,20]$. These previous findings may help determine why there was no significant difference in circulating TNF- $\alpha$ levels between severe and non-severe COVID-19 cases, thus suggesting that TNF- $\alpha$ might not have the potential as a useful biomarker for COVID-19 severity. From a clinical standpoint, it is conceivable that understanding early on which patients deteriorate and identifying biomarkers would enable heightened vigilance of these at-risk individuals.

The current meta-analysis inevitably had some inherent limitations, which need to be taken into account. The most notable drawback is the fact that most included studies are observational in design with a relatively small number of participants, which may prevent the determination of causal relationships and result in overestimation of the overall effect. Another caveat is the lack of baseline adjusted data that may limit the generalizability of our results. Furthermore, information regarding COVID-19 treatment, duration of COVID-19 infection, dietary intake, body mass index, family history, and genetic polymorphism was not available from all studies and not included in our meta-analysis, which may contribute to the presence of heterogeneity. Additionally, this meta-analysis was limited to a determination on circulating levels of other cytokines reportedly implicated in SARS-CoV-2 infection including IL-1 $\beta$, IL-2, IL-7, IL-8, and granulocyte colonystimulating factor [20,32], which might be additional contributors to ADRS in COVID-19 patients. Along with this, we did not examine circulating levels of IL-6, IL-10, and TNF- $\alpha$ in multisystem inflammatory syndrome in children (MIS-C) in the context of COVID-19. It is worth noting that future studies should be focused on the relationships between alternative cytokines known to be involved in ADRS and the disease severity and mortality in COVID-19 patients and in the patients with MIS-C, which might be helpful for a better understanding of COVID-19 etiology. Despite the limitations, there are several methodological strengths of the present meta-analysis. To our knowledge, this is the most comprehensive systematic review and meta-analysis on the relationships between circulating levels of inflammatory cytokines including IL-6, IL-10, and TNF- $\alpha$, and the severity and mortality of COVID19. Another strength point also can be observed in our extensive literature search, in which we estimated the numerical data (mean and SD) from a validated equation (when possible) to avoid losing the essential data for meta-analysis. Alongside, meta-regression, subgroup, and sensitivity analyses were executed to validate the reliability and robustness of our results.

In summary, the current systematic review with metaanalysis provided the most comprehensive empirical evidence of the significant associations of increased circulating levels of inflammatory cytokines including IL-6 and IL-10 with poor clinical outcomes and reduced survival rates in COVID-19 patients. For corroborating our findings, further longitudinal studies in different settings are needed. In parallel with this, experimental studies investigating the effects of both IL-6 and IL-10 antagonists against the developmental and progressive COVID-19 can help us better understand the therapeutic significance of cytokine storm in COVID-19, which would provide new insights into the possible therapeutic targets for COVID-19.

Acknowledgments The authors sincerely thank Ms. Saowalak Turongkaravee for her kind assistance with statistical analysis.

Authorship Contributions W.U. conceived and designed the study. W.U. and J.J. performed the systematic literature search, quality assessment, and meta-analyses. W.U. and J.J. participated in the interpretation of the results. W.U. drafted the initial manuscript. W.U., J.J., S.S., and U.C. reviewed the manuscript. W.U. critically edited and revised the 
manuscript. All authors approved the final version of the manuscript as submitted.

\section{Compliance with Ethical Standards}

Conflict of Interest The authors declare that they have no conflict of interest.

\section{References}

1. WHO Novel Coronavirus-China. Available online: https://www. who.int/csr/don/12-january-2020-novelcoronavirus-china/en/. Accessed on 20 Apr 2020.

2. World Health O. Novel coronavirus (2019-nCoV): situation report, 91. Geneva: World Health Organization; 2020.

3. Huang C, Wang Y, Li X, Ren L, Zhao J, Hu Y, et al. Clinical features of patients infected with 2019 novel coronavirus in Wuhan, China. Lancet. 2020;395:497-506.

4. Fu B, Xu X, Wei H. Why tocilizumab could be an effective treatment for severe COVID-19? J Transl Med. 2020;18:164.

5. Coperchini F, Chiovato L, Croce L, Magri F, Rotondi M. The cytokine storm in COVID-19: an overview of the involvement of the chemokine/chemokine-receptor system. Cytokine Growth Factor Rev. 2020;53:25-32.

6. Buonaguro FM, Puzanov I, Ascierto PA. Anti-IL6R role in treatment of COVID-19-related ARDS. J Transl Med. 2020;18:165.

7. McGonagle D, Sharif K, O'Regan A, Bridgewood C. The role of cytokines including interleukin-6 in COVID-19 induced pneumonia and macrophage activation syndrome-like disease. Autoimmun Rev. 2020;19:102537.

8. Hunter CA, Jones SA. IL-6 as a keystone cytokine in health and disease. Nat Immunol. 2015;16:448-57.

9. Wei H, Li B, Sun A, Guo F. Interleukin-10 family cytokines immunobiology and structure. Adv Exp Med Biol. 2019;1172: 79-96.

10. Burian E, Jungmann F, Kaissis GA, Lohöfer FK, Spinner CD, Lahmer T, et al. Intensive care risk estimation in COVID-19 pneumonia based on clinical and imaging parameters: experiences from the Munich cohort. J Clin Med. 2020;9:1514.

11. Chen X, Zhao B, Qu Y, et al. Detectable serum SARS-CoV-2 viral load (RNAaemia) is closely correlated with drastically elevated interleukin 6 (IL-6) level in critically ill COVID-19 patients. Clin Infect Dis. 2020:ciaa449. https://doi.org/10.1093/cid/ciaa449.

12. Chi Y, Ge Y, Wu B, et al. Serum cytokine and chemokine profile in relation to the severity of coronavirus disease 2019 (COVID-19) in China. J Infect Dis. 2020:jiaa363. https://doi.org/10.1093/infdis/ jiaa363.

13. Gao Y, Li T, Han M, Li X, Wu D, Xu Y, et al. Diagnostic utility of clinical laboratory data determinations for patients with the severe COVID-19. J Med Virol. 2020;92:791-6.

14. Han H, Ma Q, Li C, Liu R, Zhao L, Wang W, et al. Profiling serum cytokines in COVID-19 patients reveals IL-6 and IL-10 are disease severity predictors. Emerg Microbes Infect. 2020;9:1123-30.

15. He S, Zhou C, Lu D, Yang H, Xu H, Wu G, et al. Relationship between chest $\mathrm{CT}$ manifestations and immune response in COVID19 patients. Int J Infect Dis. 2020;98:125-9.

16. Liu L, Gao JY, Hu W, et al. Clinical characteristics of 51 patients discharged from hospital with COVID-19 in Chongqing, China. bioRxiv 2020. https://doi.org/10.1101/2020.02.20.20025536.

17. Liu T, Zhang J, Yang Y, et al. The role of interleukin-6 in monitoring severe case of coronavirus disease 2019. EMBO Mol Med. 2020;12:e12421.
18. Lv Z, Cheng S, Le J, Huang J, Feng L, Zhang B, et al. Clinical characteristics and co-infections of 354 hospitalized patients with COVID-19 in Wuhan, China: a retrospective cohort study. Microbes Infect. 2020;22:195-9.

19. Ma J, Yin J, Qian Y, Wu Y. Clinical characteristics and prognosis in cancer patients with COVID-19: a single center's retrospective study. J Inf Secur. 2020;81:318-56.

20. Qin C, Zhou L, Hu Z, Zhang S, Yang S, Tao Y, et al. Dysregulation of immune response in patients with coronavirus 2019 (COVID-19) in Wuhan, China. Clin Infect Dis. 2020;71:762-8.

21. Wan S, Yi Q, Fan S, Lv J, Zhang X, Guo L, et al. Relationships among lymphocyte subsets, cytokines, and the pulmonary inflammation index in coronavirus (COVID-19) infected patients. Br J Haematol. 2020;189:428-37.

22. Wang C, Fei D, Li X, Zhao M, Yu K. IL-6 may be a good biomarker for earlier detection of COVID-19 progression. Intensive Care Med. 2020;46:1475-6.

23. Wang H, Luo S, Shen Y, Li M, Zhang Z, Dong Y, et al. Multiple enzyme release, inflammation storm and hypercoagulability are prominent indicators for disease progression in COVID-19: a multi-centered, correlation study with CT imaging score. SSRN. 2020. https://doi.org/10.2139/ssrn.3544837.

24. Wang Y, Zhu F, Wang C, Wu J, Liu J, Chen X, et al. Children hospitalized with severe COVID-19 in Wuhan. Pediatr Infect Dis J. 2020;39:e91-4.

25. $\mathrm{Wu} \mathrm{C}, \mathrm{Hu} \mathrm{X}$, Song J, et al. Heart injury signs are associated with higher and earlier mortality in coronavirus disease 2019 (COVID19). bioRxiv 2020. https://doi.org/10.1101/2020.02.26.20028589.

26. Xie Y, You Q, Wu C, Cao S, Qu G, Yan X, et al. Impact of cardiovascular disease on clinical characteristics and outcomes of coronavirus disease 2019 (COVID-19). Circ J. 2020;84:1277-83.

27. Crespo M, Perez-Saez MJ, Redondo-Pachón D, et al. COVID-19 in elderly kidney transplant recipients. Am J Transplant. 2020: 10.1111/ajt.16096. https://doi.org/10.1111/ajt.16096.

28. Huang $\mathrm{H}$, Zhang $\mathrm{M}$, Chen $\mathrm{C}$, et al. Clinical characteristics of COVID-19 in patients with pre-existing ILD: a retrospective study in a single center in Wuhan, China. J Med Virol. 2020;13:10.1002/ jmv.26174. https://doi.org/10.1002/jmv.26174.

29. Luo M, Liu J, Jiang W, Yue S, Liu H, Wei S. IL-6 and CD8+ T cell counts combined are an early predictor of in-hospital mortality of patients with COVID-19. JCI Insight. 2020;5:139024.

30. Mikami T, Miyashita H, Yamada T, Harrington M, Steinberg D, Dunn A, et al. Risk factors for mortality in patients with COVID-19 in New York City. J Gen Intern Med. 2020:1-10. https://doi.org/10. 1007/s11606-020-05983-Z.

31. Quartuccio L, Sonaglia A, Pecori D, et al. Higher levels of IL-6 early after tocilizumab distinguish survivors from nonsurvivors in COVID-19 pneumonia: a possible indication for deeper targeting of IL-6. J Med Virol. 2020:10.1002/jmv.26149. https://doi.org/10. 1002/jmv.26149.

32. Tu WJ, Cao J, Yu L, Hu X, Liu Q. Clinicolaboratory study of 25 fatal cases of COVID-19 in Wuhan. Intensive Care Med. 2020;46: 1117-20. https://doi.org/10.1007/s00134-020-06023-4.

33. Zhou F, Yu T, Du R, et al. Clinical course and risk factors for mortality of adult inpatients with COVID-19 in Wuhan, China: a retrospective cohort study. Lancet. 2020;395:1054-62. https://doi. org/10.1016/S0140-6736(20)30566-3.

34. Zhu J, Pang J, Ji P, Zhong Z, Li H, Li B, et al. Elevated interleukin-6 is associated with severity of COVID-19: a meta-analysis. J Med Virol. 2020:10.1002/jmv.26085. https://doi.org/10.1002/jmv. 26085.

35. Higgins JPT, Thomas J, Chandler J, et al. (editors) Cochrane Handbook for Systematic Reviews of Interventions version 6.0 (updated July 2019) Cochrane, 2019. 2019. www.training. cochrane.org/handbook. Accessed 5 Jun 2020. 
36. Moher D, Liberati A, Tetzlaff J, Altman DG, The PRISMA Group. Preferred reporting items for systematic reviews and meta-analyses: the PRISMA statement. PLoS Med. 2009;6:e1000097.

37. Wells GA, Shea B, O'Connell D, et al. The NewcastleOttawa Scale (NOS) for assessing the quality of nonrandomised studies in metaanalyses. Ottawa Hospital Reserach Institute Web site; Available at: http://www.ohri.ca/programs/clinical_epidemiology/oxford.asp. Accessed 25 March 2015.

38. Luo D, Wan X, Liu J, Tong T. Optimally estimating the sample mean from the sample size, median, mid-range, and/or mid-quartile range. Stat Methods Med Res. 2018;27:1785-805.

39. Wan X, Wang W, Liu J, Tong T. Estimating the sample mean and standard deviation from the sample size, median, range and/or interquartile range. BMC Med Res Methodol. 2014;14:135.

40. Higgins JP, Thompson SG, Deeks JJ, Altman DG. Measuring inconsistency in meta-analyses. BMJ. 2003;327:557-60.
41. Bouayad A. Innate immune evasion by SARS-CoV-2: comparison with SARS-CoV. Rev Med Virol. 2020:e2135. https://doi.org/10. 1002/rmv.2135.

42. Okamoto M, Tsukamoto H, Kouwaki T, Seya T, Oshiumi H. Recognition of viral RNA by pattern recognition receptors in the induction of innate immunity and excessive inflammation during respiratory viral infections. Viral Immunol. 2017;30:408-20.

43. Shimizu M. Clinical features of cytokine storm syndrome. In: Cron R, Behrens E, editors. Cytokine storm syndrome. Cham: Springer; 2019. p. 31-42. https://doi.org/10.1007/978-3-030-22094-5_3.

Publisher's Note Springer Nature remains neutral with regard to jurisdictional claims in published maps and institutional affiliations. 